\title{
Charte du professionnalisme médical
}

\section{Préambule}

Le professionnalisme est à la base du contrat conclu entre la médecine et la société

Il exige de faire passer les intérêts des patients avant ceux du médecin, de fixer et de maintenir des normes rigoureuses de compétence et d'intégrité et de prodiguer des conseils d'expert à la société sur les questions de santé. Les principes et les responsabilités du professionnalisme médical doivent être clairement compris par la profession et par la société. Au cœur de ce contrat se situe la confiance du public dans les médecins, laquelle dépend de l'intégrité à la fois de chaque médecin et de l'ensemble de la profession.

Al'heure actuelle, la profession médicale doit affronter une explosion technologique, une évolution des forces du marché, des problèmes dans la prestation des soins de santé, le bioterrorisme et la mondialisation. Il s'ensuit que les médecins ont de plus en plus de difficulté à s'acquitter de leurs responsabilités à l'égard des patients et de la société. Dans les circonstances, il est plus important que jamais de réaffirmer les valeurs et les principes fondamentaux et universels du professionnalisme médical, qui demeurent des idéaux auxquels doivent aspirer tous les médecins.

Partout, la profession médicale est enchâssée dans des cultures et des traditions nationales diverses, mais ses membres assument tous le rôle de guérisseur, dont les racines remontent à Hippocrate. A vrai dire, la profession médicale est aux prises avec des forces politiques, juridiques et commerciales complexes. De plus, il y a de grandes divergences dans la prestation des soins et l'exercice de la médecine en vertu desquelles les principes généraux peuvent s'exprimer de manière à la fois complexe et subtile. En dépit de ces différences, des thèmes communs se dégagent et constituent le fondement de la présente charte sous forme de trois principes fondamentaux et d'un ensemble de responsabilités professionnelles décisives.

\section{Principes fondamentaux}

\section{Principe de la primauté du bien-être des patients}

Ce principe repose sur la volonté de servir les intérêts du patient. L'altruisme contribue à la confiance qui est essentielle au rapport médecin- patient. Les forces du marché, les pressions de la société et les exigences administratives ne doivent en aucun cas compromettre ce principe.

\section{Principe de I'autonomie des patients}

Les médecins doivent respecter l'autonomie des patients. Ils doivent être honnêtes avec ceux-ci et les habiliter à prendre des décisions avisées sur leur traitement. Les décisions des patients sur leurs soins doivent être d'une suprême importance, sous réserve qu'elles soient conformes à la déontologie et qu'elles ne donnent pas lieu à des demandes de soins inadaptés.

\section{Principe de la justice sociale}

La profession médicale doit promouvoir la justice dans le système de soins de santé, notamment en assurant la répartition équitable des ressources destinées aux soins de santé. Les médecins doivent s'évertuer à éliminer toute discrimination dans les soins de santé, que celle-ci soit fondée sur la race, le sexe, la situation socio-économique, l'ethnicité, la religion ou toute autre catégorie sociale.

\section{Un ensemble de responsabilités professionnelles}

\section{Engagement envers la compétence professionnelle}

Les médecins doivent s'engager à s'instruire tout au long de la vie et sont tenus d'actualiser leurs connaissances médicales et les techniques cliniques et d'équipe nécessaires à la prestation de soins de qualité. Plus généralement, la profession dans son ensemble doit faire en sorte que tous ses membres soient compétents et s'assurer que les moyens voulus sont mis à la disposition des médecins pour atteindre cet objectif.

\section{Engagement envers I'honnêteté à l'égard des patients}

Les médecins doivent fournir des renseignements exhaustifs et honnêtes aux patients avant que ceux-ci ne consentent à un traitement et à l'issue du traitement. Cela ne veut pas dire que les patients doivent prendre part à chaque décision sans exception sur les soins médicaux; ils doivent en revanche être en mesure de décider du mode de traitement. Les médecins doivent également reconnaître que dans les soins de santé, des erreurs médicales qui portent préju- 
dice aux patients sont parfois commises. Chaque fois qu'un patient subit des lésions à la suite de soins médicaux, il doit en être avisé le plus vite possible car à défaut d'agir ainsi, on compromet sérieusement la confiance du patient et de la société. La déclaration et l'analyse des erreurs médicales sont à la base de stratégies judicieuses de prévention et d'amélioration et d'un dédommagement approprié des parties lésées.

\section{Engagement envers la confidentialité des patients}

Pour mériter la confiance des patients, il faut mettre en place un dispositif de confidentialité en ce qui concerne la divulgation de renseignements sur les patients. Cet engagement s'applique aux entretiens avec les personnes qui agissent pour le compte d'un patient lorsqu'il est impossible d'obtenir le consentement du patient proprement dit. Le respect de cet engagement envers la confidentialité est plus urgent aujourd'hui que jamais, compte tenu de l'utilisation généralisée des systèmes informatiques pour remplir des données sur les patients et de la plus grande facilité d'accès aux données génétiques. Les médecins reconnaissent toutefois que leur engagement envers la confidentialité des patients doit à l'occasion céder la priorité à des considérations supérieures dans l'intérêt public (par exemple lorsque des patients mettent la vie d'autrui en péril).

\section{Engagement envers l'entretien de rapports convenants avec les patients} Etant donné la vulnérabilité inhérente et la dépendance des patients, certains rapports entre médecins et patients doivent à tout prix être évités. En particulier, un médecin ne doit jamais exploiter un patient dans le but d'en tirer un avantage sexuel, un gain financier personnel ou un autre avantage personnel.

\section{Engagement envers I'amélioration de la qualité des soins}

Les médecins doivent prendre l'engagement de constamment améliorer la qualité des soins de santé. Cet engagement ne présuppose pas seulement le maintien des compétences cliniques, mais également l'obligation de collaborer avec d'autres professionnels pour réduire les erreurs médicales, rehausser la sécurité des patients, minimiser la surutilisation des ressources des soins de santé et optimiser les résultats des soins. Les médecins doivent participer à l'élaboration de meilleures mesures de qualité des soins et à l'application de mesures de qualité pour évaluer systématiquement le rendement de tous les sujets, des établissements et des systèmes chargés de prodiguer des soins de santé. Les médecins, individuellement et par le biais de leurs associations professionnelles, doivent contribuer à la création et à l'adoption de mécanismes dont le but est de favoriser l'amélioration constante de la qualité des soins.

\section{Engagement envers I'amélioration de l'accès aux soins}

Le professionnalisme médical exige que l'objectif de tous les systèmes de soins de santé soit l'existence d'une norme de soins uniforme et suffisante. Les médecins doivent, individuellement et collectivement, s'évertuer à éliminer les entraves à des soins de santé équitables. Dans chaque système, le médecin doit s'employer à éliminer les obstacles à l'accès fondés sur l'instruction, les lois, les finances, la géographie et la discrimination sociale. L'engagement envers l'équité présuppose la promotion de la santé publique et de la médecine préventive, de même que la défense des intérêts publics par chaque médecin, sans se soucier des intérêts personnels du médecin ou de la profession médicale.

\section{Engagement envers la juste répartition de ressources limitées}

Tout en répondant aux besoins de chaque patient, les médecins sont tenus de fournir des soins de santé fondés sur la gestion prudente et rentable des moyens cliniques limités. Ils doivent prendre l'engagement de collaborer avec d'autres médecins, hôpitaux et organismes subventionnaires à l'élaboration de lignes directrices sur la rentabilité des soins. La responsabilité professionnelle du médecin à l'égard de la juste répartition des ressources exige de sa part qu'il évite scrupuleusement les examens et les procédures superflus. La prestation de services inutiles n'expose pas seulement les patients à des préjudices et à des frais évitables, mais amenuise les ressources accessibles à d'autres.

\section{Engagement envers la connaissance scientifique}

Une bonne partie du contrat entre la médecine et la société repose sur l'intégrité et l'utilisation opportune des connaissances scientifiques et des technologies. Les médecins ont le devoir de respecter les normes scientifiques, de promouvoir la recherche et de créer de nouvelles connaissances et d'assurer leur bonne utilisation. La profession est responsable de l'intégrité de ces connaissances, lesquelles reposent sur des preuves scientifiques et sur l'expérience des médecins. 


\section{Engagement envers le maintien de la confiance par la gestion des conflits d'intérêts}

Les professionnels de la médecine et leurs associations ont maintes occasions de compromettre leurs responsabilités professionnelles en recherchant des gains ou des avantages personnels. Ces compromis sont particulièrement menaçants lorsque les médecins cherchent à nouer des rapports personnels ou organisationnels avec des entreprises à but lucratif, notamment des fabricants d'équipements médicaux, des compagnies d'assurance et des compagnies pharmaceutiques. Les médecins ont l'obligation de reconnaître, de divulguer au grand public et de régler les conflits d'intérêts qui surviennent dans l'exercice de leurs fonctions et de leurs activités professionnelles. Les rapports entre l'industrie et les leaders d'opinion doivent être divulgués, surtout lorsque ces derniers fixent les critères qui régissent le déroulement des essais cliniques et les comptes rendus à leurs sujet, la rédaction d'éditoriaux ou de directives thérapeutiques ou les fonctions de rédacteur de revues scientifiques.

\section{Engagement envers les responsabilités professionnelles}

En tant que membres d'une profession, les médecins sont censés collaborer entre eux pour optimiser les soins des patients, faire preuve de respect l'un pour l'autre, et s'autoréglementer, notamment en prenant des recours et des mesures disciplinaires à l'encontre des membres qui n'ont pas respecté les normes professionnelles. La profession doit également définir et structurer le processus d'enseignement et d'établisse- ment de normes pour ses membres actuels et futurs. Les médecins sont investis d'obligations individuelles et collectives en ce qui concerne la participation à ces procédés. Ils doivent notamment se livrer à des évaluations internes et accepter l'évaluation par des gens de l'extérieur de tous les paramètres de leur rendement professionnel.

\section{Conclusion}

L'exercice de la médecine à l'époque moderne est hérissé de défis sans précédent dans pratiquement toutes les cultures et les sociétés. Ces défis s'articulent autour des disparités croissantes entre les besoins légitimes des patients, les ressources qui existent pour y répondre, la dépendance croissante à l'égard des forces du marché pour transformer les systèmes de soins de santé, et la tentation pour les médecins de ne pas tenir leur engagement traditionnel envers la primauté des intérêts des patients. Afin de préserver la fidélité du contrat social de la médecine à une époque aussi turbulente, nous sommes d'avis que les médecins doivent réaffirmer leur engagement envers les principes du professionnalisme, ce qui n'englobe pas seulement leur engagement personnel envers le bien-être de leurs patients, mais également les efforts collectifs visant à améliorer le système des soins de santé pour le bien-être de la société. Cette Charte du professionnalisme médical vise précisément à stimuler ce genre de dévouement et à promouvoir un programme d'action pour la profession médicale dont la portée et le but sont universels. 